\title{
Some inequalities for Csiszár divergence via theory of time scales
}

\author{
Iqrar Ansari1*, Khuram Ali Khan ${ }^{1}$, Ammara Nosheen², Đilda Pečarić ${ }^{3}$ and Josip Pečarić ${ }^{4}$
}

\section{"Correspondence:}

iqrar@math.qau.edu.pk

'Department of Mathematics, University of Sargodha, Sargodha, Pakistan

Full list of author information is available at the end of the article

\begin{abstract}
In this paper, we present some inequalities for Csiszár $f$-divergence between two probability measures on time scale. These results extend some known results in the literature and offer new results in $h$-discrete calculus and quantum calculus. We also present several inequalities for divergence measures.
\end{abstract}

Keywords: Csiszár divergence; Time scales calculus; Quantum calculus; Convex function

\section{Introduction}

In many applications of probability theory the essential problem is determining an appropriate measure of distance (or divergence) among two probability distributions. Consequently, many different divergence measures were introduced and extensively studied by various authors, for instance, the Csiszár $f$-divergence (Kullback-Leibler divergence, Hellinger distance, and total-variation distance), Rényi divergence, and Jensen-Shannon divergence; see $[9,13,18,20]$.

Csiszár [6] introduced the following:

Definition 1 Let $f: \mathbb{R}^{+} \rightarrow \mathbb{R}^{+}$be a convex function. Let $\tilde{\mathbf{r}}=\left(r_{1}, r_{2}, \ldots, r_{n}\right)$ and $\tilde{\mathbf{s}}=$ $\left(s_{1}, s_{2}, \ldots, s_{n}\right)$ be such that $\sum_{v=1}^{n} r_{v}=1$ and $\sum_{v=1}^{n} s_{v}=1$. Then the $f$-divergence functional is defined as

$$
I_{f}(\tilde{\mathbf{r}}, \tilde{\mathbf{s}}):=\sum_{\nu=1}^{n} s_{v} f\left(\frac{r_{v}}{s_{v}}\right)
$$

where $f$ satisfies the following conditions:

$$
f(0):=\lim _{\theta \rightarrow 0^{+}} f(\theta) ; \quad 0 f\left(\frac{0}{0}\right):=0 ; \quad 0 f\left(\frac{a}{0}\right):=\lim _{\theta \rightarrow 0^{+}} \theta f\left(\frac{a}{0}\right), \quad a>0 .
$$

Dragomir $[7,8]$ has done a plenty of work giving different types of bounds on the distance and divergence measures. Jensen's inequality plays a vital role to get inequalities for divergences between probability distributions. Horvath et al. [11] introduced a new

(c) The Author(s) 2020. This article is licensed under a Creative Commons Attribution 4.0 International License, which permits use, sharing, adaptation, distribution and reproduction in any medium or format, as long as you give appropriate credit to the original author(s) and the source, provide a link to the Creative Commons licence, and indicate if changes were made. The images or other third party material in this article are included in the article's Creative Commons licence, unless indicated otherwise in a credit line to the material. If material is not included in the article's Creative Commons licence and your intended use is not permitted by statutory regulation or exceeds the permitted use, you will need to obtain permission directly from the copyright holder. To view a copy of this licence, visit http://creativecommons.org/licenses/by/4.0/. 
functional based on the $f$-divergence functional and obtained some estimates for the new functional, the $f$-divergence and Rényi divergence by utilizing cyclic refinement of Jensen's inequality. Recently, Adil et al. [12] obtained some inequalities for convex functions and their applications to Csiszár divergence.

The main objective behind the theory of time scales is unifying continuous and discrete analysis introduced by Stefan Hilger in 1988 and established in the comprehensive books $[4,5]$. Various dynamic derivatives on time scales not just give a helpful route in useful applications, but also demonstrate their extraordinary appearance in approximations. It may be beneficial to examine if such useful features can be kept up or even improved in a specific way while different dynamic derivatives are utilized in the same application simultaneously.

Guseinov [10] examined the process of Riemann and Lebesgue integration on time scales. Many authors established time scale version of linear and nonlinear integral inequalities $[1,17,19]$. The time scale integral inequalities have been used to study the boundedness, uniqueness, and so on of the solutions of different dynamic equations $[14,16]$. Ansari et al. [2] introduced the differential entropy of a continuous random variable on time scales and established some Shannon-type inequalities on arbitrary time scales. It was shown that the obtained inequalities are used to estimate the bounds of differential entropy for some particular distributions. Some classical inequalities and their converses for multiple integration on time scales were investigated in [3].

The setup of this paper is as follows. Section 2 is confined to the basic definitions and preliminary results of time scales calculus. Our aim in Sect. 3 is deriving some new inequalities for Csiszár $f$-divergence on arbitrary time scales and finding some inequalities for Csiszár divergence in $h$-discrete calculus and quantum calculus. To the best of the author's knowledge, no contribution is available in the literature for Csiszár divergence inequalities in quantum calculus. Section 4 is concerned to the study of some divergence measures on time scales including the bounds of the Kullback-Leibler distance, triangular discrimination, Hellinger discrimination, Jeffreys distance, Bhattacharyya distance, and harmonic distance in terms of some special means such as identric, logarithmic, arithmetic, and geometric means. The upper bounds of these divergence results in quantum calculus are also part of discussion.

\section{Preliminaries}

In this paper, we assume that a time scale $\mathbb{T}$ is an arbitrary nonempty closed subset of the real line. The following definitions and results are extracted from [4].

Definition 2 Consider a time scale $\mathbb{T}$ that is a closed and bounded subset of real numbers and $\omega \in \mathbb{T}$. Then the mappings $\sigma: \mathbb{T} \rightarrow \mathbb{T}$ and $\rho: \mathbb{T} \rightarrow \mathbb{T}$ satisfying

$$
\sigma(\omega)=\inf \{\lambda \in \mathbb{T}: \lambda>\omega\} \quad \text { and } \quad \rho(\omega)=\sup \{\lambda \in \mathbb{T}: \lambda<\omega\}
$$

are known as forward and backward jump operators on $\mathbb{T}$, respectively.

A function $z: \mathbb{T} \rightarrow \mathbb{R}$ is right-dense continuous or rd-continuous if it is continuous at right-dense points in $\mathbb{T}$ and its left-sided limits exist (finite) at left-dense points in $\mathbb{T}$. The set of all rd-continuous functions is denoted by $C_{\mathrm{rd}}$. 
Here we define $\mathbb{T}^{k}$ as follows:

$$
\mathbb{T}^{k}= \begin{cases}\mathbb{T} \backslash(\rho(\sup \mathbb{T}), \sup \mathbb{T}] & \text { if } \sup \mathbb{T}<\infty \\ \mathbb{T} & \text { if } \sup \mathbb{T}=\infty\end{cases}
$$

Definition 3 Let $z: \mathbb{T} \rightarrow \mathbb{R}$ and $\omega \in \mathbb{T}^{k}$. Then we define the delta derivative $z^{\Delta}(\omega)$ as the number (provided it exists) such that for each $\epsilon>0$, there exists a neighborhood $U$ of $\omega$ such that

$$
\left|z(\sigma(\omega))-z(\lambda)-z^{\Delta}(\omega)(\sigma(\omega)-\lambda)\right| \leq \epsilon|\sigma(\omega)-\lambda|
$$

for all $\lambda \in U$. We say that $z$ is delta differentiable at $\omega$.

If $\mathbb{T}=\mathbb{R}$, then $z^{\Delta}$ is the the usual derivative $z^{\prime}$, whereas $z^{\Delta}$ becomes the forward difference operator $\Delta z(\omega)=z(\omega+1)-z(\omega)$ for $\mathbb{T}=\mathbb{Z}$. If $\mathbb{T}=\overline{q^{\mathbb{Z}}}=\left\{q^{n}: n \in \mathbb{Z}\right\} \cup\{0\}$ with $q>1$, then $z^{\Delta}$ is the so-called $q$-difference operator

$$
z^{\Delta}(\omega)=\frac{z(q \omega)-z(\omega)}{(q-1) \omega}, \quad z^{\Delta}(0)=\lim _{\lambda \rightarrow 0} \frac{z(\lambda)-z(0)}{\lambda} .
$$

Theorem 1 (Existence of antiderivatives) Every rd-continuous function has an antiderivative. In particular if $x_{0} \in \mathbb{T}$, then $F$ is defined by

$$
F(x):=\int_{x_{0}}^{x} f(\omega) \Delta \omega \quad \text { for } x \in \mathbb{T}^{k}
$$

which is an antiderivative of $f$.

For $\mathbb{T}=\mathbb{R}$, we get $\int_{a}^{b} z(\omega) \Delta \omega=\int_{a}^{b} z(\omega) d \omega$, and if $\mathbb{T}=\mathbb{N}$, then $\int_{a}^{b} z(\omega) \Delta \omega=\sum_{\omega=a}^{b-1} z(\omega)$, where $a, b \in \mathbb{T}$ with $a \leq b$.

\section{Main results}

Let $\mathbb{T}$ be a time scale and consider the set of all probability density functions on $\mathbb{T}$,

$$
\Omega:=\left\{\tilde{r} \in C_{\mathrm{rd}}\left([a, b]_{\mathbb{T}},[0, \infty)\right), \tilde{r}(x) \geq 0, \int_{a}^{b} \tilde{r}(x) \Delta x=1\right\}
$$

In this paper, we assume that $\tilde{r}, \tilde{s} \in \Omega$.

Definition 4 The Csiszár $f$-divergence on time scales is defined as

$$
D_{f}(\tilde{s}, \tilde{r}):=\int_{a}^{b} \tilde{r}(x) f\left(\frac{\tilde{s}(x)}{\tilde{r}(x)}\right) \Delta x
$$

where $f$ is convex on $(0, \infty)$.

By suitable substitutions to $f$ in Definition 4 we can obtain several divergences on time scales. For instance, if we choose $f(x)=x^{2}-1$, then we find the Pearson $\chi^{2}$-divergence on 
time scales denoted by $D_{\chi^{2}}$ and defined as

$$
D_{\chi^{2}}(\tilde{s}, \tilde{r}):=\int_{a}^{b} \tilde{r}(x)\left[\left(\frac{\tilde{s}(x)}{\tilde{r}(x)}\right)^{2}-1\right] \Delta x .
$$

We begin with the following result.

Theorem 2 Let $\psi:[0, \infty) \rightarrow \mathbb{R}$ be a mapping convex on the interval $\left[\zeta_{1}, \zeta_{2}\right] \subset[0, \infty)$, where $\zeta_{1} \leq 1 \leq \zeta_{2}$. If

$$
\zeta_{1} \leq \frac{\tilde{r}(y)}{\tilde{s}(y)} \leq \zeta_{2} \quad \text { for all } y \in \mathbb{T},
$$

then

$$
I_{\psi}(\tilde{r}, \tilde{s})=\int_{a}^{b} \tilde{s}(y) \psi\left(\frac{\tilde{r}(y)}{\tilde{s}(y)}\right) \Delta y \leq \frac{\zeta_{2}-1}{\zeta_{2}-\zeta_{1}} \psi\left(\zeta_{1}\right)+\frac{1-\zeta_{1}}{\zeta_{2}-\zeta_{1}} \psi\left(\zeta_{2}\right)
$$

Proof As $\psi$ is convex on $\left[\zeta_{1}, \zeta_{2}\right]$, we can write

$$
\psi\left(t \zeta_{1}+(1-t) \zeta_{2}\right) \leq t \psi\left(\zeta_{1}\right)+(1-t) \psi\left(\zeta_{2}\right) \quad \text { for all } t \in[0,1] .
$$

Choose $t=\frac{\zeta_{2}-x}{\zeta_{2}-\zeta_{1}}, x \in\left[\zeta_{1}, \zeta_{2}\right]$. Then $1-t=\frac{x-\zeta_{1}}{\zeta_{2}-\zeta_{1}}$, and from (2) we get

$$
\psi(x) \leq \frac{\zeta_{2}-x}{\zeta_{2}-\zeta_{1}} \psi\left(\zeta_{1}\right)+\frac{x-\zeta_{1}}{\zeta_{2}-\zeta_{1}} \psi\left(\zeta_{2}\right) \quad \text { for all } x \in\left[\zeta_{1}, \zeta_{2}\right]
$$

Using $x=\frac{\tilde{r}(y)}{\tilde{s}(y)}, y \in \mathbb{T}$, in (3), we get

$$
\psi\left(\frac{\tilde{r}(y)}{\tilde{s}(y)}\right) \leq \frac{\zeta_{2}-\frac{\tilde{r}(y)}{\tilde{s}(y)}}{\zeta_{2}-\zeta_{1}} \psi\left(\zeta_{1}\right)+\frac{\frac{\tilde{r}(y)}{\tilde{s}(y)}-\zeta_{1}}{\zeta_{2}-\zeta_{1}} \psi\left(\zeta_{2}\right) \quad \text { for all } y \in \mathbb{T}
$$

Multiplying (4) by $\tilde{s}(y)>0$, integrating over $\mathbb{T}$, and using the equalities $\int_{a}^{b} \tilde{r}(y) \Delta y=$ $\int_{a}^{b} \tilde{s}(y) \Delta y=1$, we obtain

$$
I_{\psi}(\tilde{r}, \tilde{s})=\int_{a}^{b} \tilde{s}(y) \psi\left(\frac{\tilde{r}(y)}{\tilde{s}(y)}\right) \Delta y \leq \frac{\zeta_{2}-1}{\zeta_{2}-\zeta_{1}} \psi\left(\zeta_{1}\right)+\frac{1-\zeta_{1}}{\zeta_{2}-\zeta_{1}} \psi\left(\zeta_{2}\right)
$$

which is the stated result.

Example 1 For $\mathbb{T}=\mathbb{R}$, Theorem 2 becomes [7, Theorem 1 on p. 2].

Example 2 Choosing $\mathbb{T}=h \mathbb{Z}, h>0$, in Theorem 2, we get

$$
\sum_{k=\frac{a}{h}}^{\frac{b}{h}-1} \tilde{s}(k h) h \psi\left(\frac{\tilde{r}(k h)}{\tilde{s}(k h)}\right) \leq \frac{\zeta_{2}-1}{\zeta_{2}-\zeta_{1}} \psi\left(\zeta_{1}\right)+\frac{1-\zeta_{1}}{\zeta_{2}-\zeta_{1}} \psi\left(\zeta_{2}\right) .
$$

Remark 1 Inequality (5) in $h$-discrete calculus is an extension of specific upper bound for the Csiszár divergence obtained by Lovričević et al. [15, Corollary 4.1]. 
Example 3 Choosing $\mathbb{T}=q^{\mathbb{N}_{0}}(q>1)$ in Theorem 2, we have

$$
\sum_{k=\log _{q}(a)}^{\log _{q}(b)-1} q^{k}(q-1) \tilde{s}\left(q^{k}\right) \psi\left(\frac{\tilde{r}\left(q^{k}\right)}{\tilde{s}\left(q^{k}\right)}\right) \leq \frac{\zeta_{2}-1}{\zeta_{2}-\zeta_{1}} \psi\left(\zeta_{1}\right)+\frac{1-\zeta_{1}}{\zeta_{2}-\zeta_{1}} \psi\left(\zeta_{2}\right)
$$

Remark 2 Equation (6) represents the Csiszár divergence in quantum calculus, which is new up to the knowledge of the authors.

Theorem 3 Consider a differentiable convex function $\psi:[0, \infty) \rightarrow \mathbb{R}$ on the interval $\left[\zeta_{1}, \zeta_{2}\right]$ and $I_{\psi}$ defined in Theorem 2 . Then we have

$$
\begin{aligned}
0 & \leq \frac{\zeta_{2}-1}{\zeta_{2}-\zeta_{1}} \psi\left(\zeta_{1}\right)+\frac{1-\zeta_{1}}{\zeta_{2}-\zeta_{1}} \psi\left(\zeta_{2}\right)-I_{\psi}(\tilde{r}, \tilde{s}) \\
& \leq \frac{\psi^{\prime}\left(\zeta_{2}\right)-\psi^{\prime}\left(\zeta_{1}\right)}{\zeta_{2}-\zeta_{1}}\left[\left(\zeta_{2}-1\right)\left(1-\zeta_{1}\right)-D_{\chi^{2}}(\tilde{r}, \tilde{s})\right] \\
& \leq \frac{1}{4}\left(\zeta_{2}-\zeta_{1}\right)\left[\psi^{\prime}\left(\zeta_{2}\right)-\psi^{\prime}\left(\zeta_{1}\right)\right]
\end{aligned}
$$

where $D_{\chi^{2}}(\tilde{r}, \tilde{s}):=\int_{a}^{b} \tilde{s}(y)\left[\left(\frac{\tilde{r}(y)}{\tilde{s}(y)}\right)^{2}-1\right] \Delta y$.

Proof Since $\psi$ is a differentiable convex function, we have

$$
\psi\left(u_{1}\right)-\psi\left(u_{2}\right) \geq \psi^{\prime}\left(u_{2}\right)\left(u_{1}-u_{2}\right) \quad \text { for all } u_{1}, u_{2} \in\left(\zeta_{1}, \zeta_{2}\right)
$$

Now assume that $a_{1}, a_{2} \in\left[\zeta_{1}, \zeta_{2}\right]$ and consider $\alpha_{1}, \alpha_{2} \geq 0$ such that $\alpha_{1}+\alpha_{2}>0$. Then using $u_{1}=\frac{\alpha_{1} a_{1}+\alpha_{2} a_{2}}{\alpha_{1}+\alpha_{2}}$ and $u_{2}=a_{1}$ in (10), we get

$$
\begin{aligned}
\psi\left(\frac{\alpha_{1} a_{1}+\alpha_{2} a_{2}}{\alpha_{1}+\alpha_{2}}\right)-\psi\left(a_{1}\right) & \geq \psi^{\prime}\left(a_{1}\right)\left(\frac{\alpha_{1} a_{1}+\alpha_{2} a_{2}}{\alpha_{1}+\alpha_{2}}-a_{1}\right) \\
& =\frac{\alpha_{2}}{\alpha_{1}+\alpha_{2}} \psi^{\prime}\left(a_{1}\right)\left(a_{2}-a_{1}\right) .
\end{aligned}
$$

Rewrite (11) with $u_{2}=a_{2}$ :

$$
\begin{aligned}
\psi\left(\frac{\alpha_{1} a_{1}+\alpha_{2} a_{2}}{\alpha_{1}+\alpha_{2}}\right)-\psi\left(a_{2}\right) & \geq \psi^{\prime}\left(a_{2}\right)\left(\frac{\alpha_{1} a_{1}+\alpha_{2} a_{2}}{\alpha_{1}+\alpha_{2}}-a_{2}\right) \\
& =-\frac{\alpha_{1}}{\alpha_{1}+\alpha_{2}} \psi^{\prime}\left(a_{2}\right)\left(a_{2}-a_{1}\right) .
\end{aligned}
$$

Multiplying (11) by $\alpha_{1}$ and (12) by $\alpha_{2}$ and then adding the resultant inequalities, we get

$$
\begin{gathered}
\left(\alpha_{1}+\alpha_{2}\right) \psi\left(\frac{\alpha_{1} a_{1}+\alpha_{2} a_{2}}{\alpha_{1}+\alpha_{2}}\right)-\alpha_{1} \psi\left(a_{1}\right)-\alpha_{2} \psi\left(a_{2}\right) \\
\geq \frac{\alpha_{1} \alpha_{2}}{\alpha_{1}+\alpha_{2}}\left(a_{2}-a_{1}\right)\left(\psi^{\prime}\left(a_{1}\right)-\psi^{\prime}\left(a_{2}\right)\right) .
\end{gathered}
$$


Dividing (13) by $-\left(\alpha_{1}+\alpha_{2}\right)$, we obtain

$$
\begin{aligned}
0 & \leq \frac{\alpha_{1} \psi\left(a_{1}\right)+\alpha_{2} \psi\left(a_{2}\right)}{\alpha_{1}+\alpha_{2}}-\psi\left(\frac{\alpha_{1} a_{1}+\alpha_{2} a_{2}}{\alpha_{1}+\alpha_{2}}\right) \\
& \leq \frac{\alpha_{1} \alpha_{2}}{\left(\alpha_{1}+\alpha_{2}\right)^{2}}\left(a_{2}-a_{1}\right)\left(\psi^{\prime}\left(a_{2}\right)-\psi^{\prime}\left(a_{1}\right)\right) .
\end{aligned}
$$

Now using $\alpha_{1}=\zeta_{2}-x, \alpha_{2}=x-\zeta_{1}, a_{1}=\zeta_{1}, a_{2}=\zeta_{2}$ in (14), we get

$$
\begin{aligned}
0 & \leq \frac{\left(\zeta_{2}-x\right) \psi\left(\zeta_{1}\right)+\left(x-\zeta_{1}\right) \psi\left(\zeta_{2}\right)}{\left(\zeta_{2}-\zeta_{1}\right)}-\psi(x) \\
& \leq \frac{\left(\zeta_{2}-x\right)\left(x-\zeta_{1}\right)}{\left(\zeta_{2}-\zeta_{1}\right)}\left(\psi^{\prime}\left(\zeta_{2}\right)-\psi^{\prime}\left(\zeta_{1}\right)\right) .
\end{aligned}
$$

Putting $x=\frac{\tilde{r}(y)}{\tilde{s}(y)}$ in $(15)$ and multiplying by $\tilde{s}(y)$, we obtain

$$
\begin{gathered}
\frac{\left(\zeta_{2} \tilde{s}(y)-\tilde{r}(y)\right) \psi\left(\zeta_{1}\right)+\left(\tilde{r}(y)-\zeta_{1} \tilde{s}(y)\right) \psi\left(\zeta_{2}\right)}{\left(\zeta_{2}-\zeta_{1}\right)}-\tilde{s}(y) \psi\left(\frac{\tilde{r}(y)}{\tilde{s}(y)}\right) \\
\leq \frac{\left(\zeta_{2} \tilde{s}(y)-\tilde{r}(y)\right)\left(\tilde{r}(y)-\zeta_{1} \tilde{s}(y)\right)}{\left(\zeta_{2}-\zeta_{1}\right) \tilde{s}(y)}\left(\psi^{\prime}\left(\zeta_{2}\right)-\psi^{\prime}\left(\zeta_{1}\right)\right)
\end{gathered}
$$

for all $y \in \mathbb{T}$.

By taking $\Delta$-integral on both sides of (16) with $\int_{a}^{b} \tilde{r}(y) \Delta y=\int_{a}^{b} \tilde{s}(y) \Delta y=1$ we get

$$
\begin{aligned}
& \frac{\left(\zeta_{2}-1\right) \psi\left(\zeta_{1}\right)+\left(1-\zeta_{1}\right) \psi\left(\zeta_{2}\right)}{\left(\zeta_{2}-\zeta_{1}\right)}-I_{\psi}(\tilde{r}, \tilde{s}) \\
\leq & \frac{\left(\psi^{\prime}\left(\zeta_{2}\right)-\psi^{\prime}\left(\zeta_{1}\right)\right)}{\left(\zeta_{2}-\zeta_{1}\right)} \int_{a}^{b} \frac{\left(\zeta_{2} \tilde{s}(y)-\tilde{r}(y)\right)\left(\tilde{r}(y)-\zeta_{1} \tilde{s}(y)\right)}{\tilde{s}(y)} \Delta y \\
= & \frac{\left(\psi^{\prime}\left(\zeta_{2}\right)-\psi^{\prime}\left(\zeta_{1}\right)\right)}{\left(\zeta_{2}-\zeta_{1}\right)}\left[\zeta_{2}-\int_{a}^{b} \frac{\tilde{r}^{2}(y)}{\tilde{s}(y)} \Delta y-\zeta_{1} \zeta_{2}+\zeta_{1}\right] \\
= & \frac{\left(\psi^{\prime}\left(\zeta_{2}\right)-\psi^{\prime}\left(\zeta_{1}\right)\right)}{\left(\zeta_{2}-\zeta_{1}\right)}\left[\zeta_{2}-\int_{a}^{b} \tilde{s}(y)\left(\frac{\tilde{r}(y)}{\tilde{s}(y)}\right)^{2} \Delta y+\int_{a}^{b} \tilde{s}(y) \Delta y\right. \\
& \left.-\int_{a}^{b} \tilde{s}(y) \Delta y-\zeta_{1} \zeta_{2}+\zeta_{1}\right] \\
= & \frac{\left(\psi^{\prime}\left(\zeta_{2}\right)-\psi^{\prime}\left(\zeta_{1}\right)\right)}{\left(\zeta_{2}-\zeta_{1}\right)}\left[\zeta_{2}+\zeta_{1}-\zeta_{1} \zeta_{2}-1-D_{\chi^{2}}(\tilde{r}, \tilde{s})\right] \\
= & \frac{\left(\psi^{\prime}\left(\zeta_{2}\right)-\psi^{\prime}\left(\zeta_{1}\right)\right)}{\left(\zeta_{2}-\zeta_{1}\right)}\left[\left(\zeta_{2}-1\right)\left(1-\zeta_{1}\right)-D_{\chi^{2}}(\tilde{r}, \tilde{s})\right],
\end{aligned}
$$

which is inequality (8). Inequality (9) is obvious, since

$$
\left(\zeta_{2}-1\right)\left(1-\zeta_{1}\right) \leq \frac{1}{4}\left(\zeta_{2}-\zeta_{1}\right)^{2} \quad \text { and } \quad D_{\chi^{2}}(\tilde{r}, \tilde{s}) \geq 0
$$

Example 4 Choosing $\mathbb{T}=\mathbb{R}$ in Theorem 3, we get [7, Theorem 2 on p. 3]. 
Example 5 Choosing $\mathbb{T}=h \mathbb{Z}, h>0$, in Theorem 3, we obtain

$$
\begin{aligned}
0 & \leq \frac{\zeta_{2}-1}{\zeta_{2}-\zeta_{1}} \psi\left(\zeta_{1}\right)+\frac{1-\zeta_{1}}{\zeta_{2}-\zeta_{1}} \psi\left(\zeta_{2}\right)-\sum_{k=\frac{a}{h}}^{\frac{b}{h}-1} \tilde{s}(k h) h \psi\left(\frac{\tilde{r}(k h)}{\tilde{s}(k h)}\right) \\
& \leq \frac{\psi^{\prime}\left(\zeta_{2}\right)-\psi^{\prime}\left(\zeta_{1}\right)}{\zeta_{2}-\zeta_{1}}\left(\left(\zeta_{2}-1\right)\left(1-\zeta_{1}\right)-\sum_{k=\frac{a}{h}}^{\frac{b}{h}-1} \tilde{s}(k h) h\left[\left(\frac{\tilde{r}(k h)}{\tilde{s}(k h)}\right)^{2}-1\right]\right) \\
& \leq \frac{1}{4}\left(\zeta_{2}-\zeta_{1}\right)\left[\psi^{\prime}\left(\zeta_{2}\right)-\psi^{\prime}\left(\zeta_{1}\right)\right] .
\end{aligned}
$$

Example 6 Choosing $\mathbb{T}=q^{\mathbb{N}_{0}}(q>1)$ in Theorem 3 , we get

$$
\begin{aligned}
0 & \leq \frac{\zeta_{2}-1}{\zeta_{2}-\zeta_{1}} \psi\left(\zeta_{1}\right)+\frac{1-\zeta_{1}}{\zeta_{2}-\zeta_{1}} \psi\left(\zeta_{2}\right)-\sum_{k=\log _{q}(a)}^{\log _{q}(b)-1} q^{k}(q-1) \tilde{s}\left(q^{k}\right) \psi\left(\frac{\tilde{r}\left(q^{k}\right)}{\tilde{s}\left(q^{k}\right)}\right) \\
& \leq \frac{\psi^{\prime}\left(\zeta_{2}\right)-\psi^{\prime}\left(\zeta_{1}\right)}{\zeta_{2}-\zeta_{1}}\left(\left(\zeta_{2}-1\right)\left(1-\zeta_{1}\right)-\sum_{k=\log _{q}(a)}^{\log _{q}(b)-1} q^{k}(q-1) \tilde{s}\left(q^{k}\right)\left[\left(\frac{\tilde{r}\left(q^{k}\right)}{\tilde{s}\left(q^{k}\right)}\right)^{2}-1\right]\right) \\
& \leq \frac{1}{4}\left(\zeta_{2}-\zeta_{1}\right)\left[\psi^{\prime}\left(\zeta_{2}\right)-\psi^{\prime}\left(\zeta_{1}\right)\right] .
\end{aligned}
$$

Remark 3 Equation (17) is new in quantum calculus, which involves the Csiszár divergence and Pearson $\chi^{2}$-divergence.

Theorem 4 Consider a twice differentiable function $\Psi:[0, \infty) \rightarrow \mathbb{R}$ on $\left[\zeta_{1}, \zeta_{2}\right]$ with

$$
m \leq \Psi^{\prime \prime}(t) \leq M \quad \text { for all } t \in\left[\zeta_{1}, \zeta_{2}\right]
$$

If $\zeta_{1} \leq \frac{\tilde{r}(y)}{\tilde{s}(y)} \leq \zeta_{2}$ for all $y \in \mathbb{T}$, then

$$
\begin{aligned}
& \frac{m}{2}\left[\left(\zeta_{2}-1\right)\left(1-\zeta_{1}\right)-D_{\chi^{2}}(\tilde{r}, \tilde{s})\right] \\
& \quad \leq \frac{\zeta_{2}-1}{\zeta_{2}-\zeta_{1}} \Psi\left(\zeta_{1}\right)+\frac{1-\zeta_{1}}{\zeta_{2}-\zeta_{1}} \Psi\left(\zeta_{2}\right)-I_{\Psi}(\tilde{r}, \tilde{s}) \\
& \quad \leq \frac{1}{2} M\left[\left(\zeta_{2}-1\right)\left(1-\zeta_{1}\right)-D_{\chi^{2}}(\tilde{r}, \tilde{s})\right]
\end{aligned}
$$

where $D_{\chi^{2}}(\tilde{r}, \tilde{s})$ is defined in Theorem 3 .

Proof Define $\eta_{m}:[0, \infty) \rightarrow \mathbb{R}$ by $\eta_{m}(t)=\Psi(t)-\frac{1}{2} m t^{2}$. Then $\eta_{m}^{\prime \prime}(t)=\Psi^{\prime \prime}(t)-m \geq 0, t \in$ $\left[\zeta_{1}, \zeta_{2}\right]$, and this implies that $\eta_{m}$ is convex on $\left[\zeta_{1}, \zeta_{2}\right]$. By using (1) for $\eta_{m}$ instead of $\psi$ we get

$$
I_{\Psi-\frac{1}{2} m(\cdot)^{2}}(\tilde{r}, \tilde{s}) \leq \frac{\zeta_{2}-1}{\zeta_{2}-\zeta_{1}}\left[\Psi\left(\zeta_{1}\right)-\frac{1}{2} m \zeta_{1}^{2}\right]+\frac{1-\zeta_{1}}{\zeta_{2}-\zeta_{1}}\left[\Psi\left(\zeta_{2}\right)-\frac{1}{2} m \zeta_{2}^{2}\right]
$$


However,

$$
\begin{aligned}
& I_{\Psi-\frac{1}{2} m(\cdot)^{2}}(\tilde{r}, \tilde{s}) \\
& \quad=I_{\Psi}(\tilde{r}, \tilde{s})-\frac{1}{2} m\left[\int_{a}^{b} \tilde{s}(y) \frac{\tilde{r}^{2}(y)}{\tilde{s}^{2}(y)} \Delta y\right] \\
& \quad=I_{\Psi}(\tilde{r}, \tilde{s})-\frac{1}{2} m\left[\int_{a}^{b} \frac{\tilde{r}^{2}(y)}{\tilde{s}(y)} \Delta y-1+1\right] \\
& \quad=I_{\Psi}(\tilde{r}, \tilde{s})-\frac{1}{2} m D_{\chi^{2}}(\tilde{r}, \tilde{s})-\frac{1}{2} m,
\end{aligned}
$$

and by (20) we obtain

$$
\begin{aligned}
& \frac{1}{2} m \zeta_{2}^{2} \frac{\left(1-\zeta_{1}\right)}{\left(\zeta_{2}-\zeta_{1}\right)}+\frac{1}{2} m \zeta_{1}^{2} \frac{\left(\zeta_{2}-1\right)}{\left(\zeta_{2}-\zeta_{1}\right)}-\frac{1}{2} m D_{\chi^{2}}(\tilde{r}, \tilde{s})-\frac{1}{2} m \\
& \quad \leq \frac{\left(\zeta_{2}-1\right)}{\zeta_{2}-\zeta_{1}} \Psi\left(\zeta_{1}\right)+\frac{\left(1-\zeta_{1}\right)}{\zeta_{2}-\zeta_{1}} \Psi\left(\zeta_{2}\right)-I_{\Psi}(\tilde{r}, \tilde{s})
\end{aligned}
$$

Simplification of the left-hand side of (21) gives

$$
\begin{aligned}
\frac{1}{2} & m \zeta_{2}^{2} \frac{\left(1-\zeta_{1}\right)}{\left(\zeta_{2}-\zeta_{1}\right)}+\frac{1}{2} m \zeta_{1}^{2} \frac{\left(\zeta_{2}-1\right)}{\left(\zeta_{2}-\zeta_{1}\right)}-\frac{1}{2} m D_{\chi^{2}}(\tilde{r}, \tilde{s})-\frac{1}{2} m \\
& =\frac{1}{2} m\left[\frac{\zeta_{2}^{2}\left(1-\zeta_{1}\right)+\zeta_{1}^{2}\left(\zeta_{2}-1\right)}{\left(\zeta_{2}-\zeta_{1}\right)}-D_{\chi^{2}}(\tilde{r}, \tilde{s})-1\right] \\
& =\frac{1}{2} m\left[\frac{\zeta_{2}^{2}-\zeta_{1} \zeta_{2}^{2}+\zeta_{1}^{2} \zeta_{2}-\zeta_{1}^{2}}{\left(\zeta_{2}-\zeta_{1}\right)}-1-D_{\chi^{2}}(\tilde{r}, \tilde{s})\right] \\
& =\frac{1}{2} m\left[\frac{\zeta_{2}^{2}-\zeta_{1}^{2}-\zeta_{1} \zeta_{2}\left(\zeta_{2}-\zeta_{1}\right)}{\left(\zeta_{2}-\zeta_{1}\right)}-1-D_{\chi^{2}}(\tilde{r}, \tilde{s})\right] \\
= & \frac{1}{2} m\left[\zeta_{2}+\zeta_{1}-\zeta_{1} \zeta_{2}-1-D_{\chi^{2}}(\tilde{r}, \tilde{s})\right] \\
= & \frac{1}{2} m\left[\left(\zeta_{2}-1\right)\left(1-\zeta_{1}\right)-D_{\chi^{2}}(\tilde{r}, \tilde{s})\right]
\end{aligned}
$$

and (18) is proved. Similarly, inequality (19) can be obtained for the mapping $\eta_{m}(t)=$ $\frac{1}{2} M t^{2}-\Psi(t)$.

Example 7 Choosing $\mathbb{T}=\mathbb{R}$ in Theorem 4, we get [7, Theorem 3 on p. 4].

Example 8 Choosing $\mathbb{T}=h \mathbb{Z}, h>0$, in Theorem 4 , we have

$$
\begin{aligned}
& \frac{m}{2}\left(\left(\zeta_{2}-1\right)\left(1-\zeta_{1}\right)-\sum_{k=\frac{a}{h}}^{\frac{b}{h}-1} \tilde{s}(k h) h\left[\left(\frac{\tilde{r}(k h)}{\tilde{s}(k h)}\right)^{2}-1\right]\right) \\
& \quad \leq \frac{\zeta_{2}-1}{\zeta_{2}-\zeta_{1}} \Psi\left(\zeta_{1}\right)+\frac{1-\zeta_{1}}{\zeta_{2}-\zeta_{1}} \Psi\left(\zeta_{2}\right)-\sum_{k=\frac{a}{h}}^{\frac{b}{h}-1} \tilde{s}(k h) h \Psi\left(\frac{\tilde{r}(k h)}{\tilde{s}(k h)}\right) \\
& \quad \leq \frac{1}{2} M\left(\left(\zeta_{2}-1\right)\left(1-\zeta_{1}\right)-\sum_{k=\frac{a}{h}}^{\frac{b}{h}-1} \tilde{s}(k h) h\left[\left(\frac{\tilde{r}(k h)}{\tilde{s}(k h)}\right)^{2}-1\right]\right) .
\end{aligned}
$$


Example 9 Choosing $\mathbb{T}=q^{\mathbb{N}_{0}}(q>1)$ in Theorem 4, we have

$$
\begin{aligned}
& \frac{m}{2}\left(\left(\zeta_{2}-1\right)\left(1-\zeta_{1}\right)-\sum_{k=\log _{q}(a)}^{\log _{q}(b)-1} q^{k}(q-1) \tilde{s}\left(q^{k}\right)\left[\left(\frac{\tilde{r}\left(q^{k}\right)}{\tilde{s}\left(q^{k}\right)}\right)^{2}-1\right]\right) \\
& \quad \leq \frac{\zeta_{2}-1}{\zeta_{2}-\zeta_{1}} \Psi\left(\zeta_{1}\right)+\frac{1-\zeta_{1}}{\zeta_{2}-\zeta_{1}} \Psi\left(\zeta_{2}\right)-\sum_{k=\log _{q}(a)}^{\log _{q}(b)-1} q^{k}(q-1) \tilde{s}\left(q^{k}\right) \Psi\left(\frac{\tilde{r}\left(q^{k}\right)}{\tilde{s}\left(q^{k}\right)}\right) \\
& \quad \leq \frac{1}{2} M\left(\left(\zeta_{2}-1\right)\left(1-\zeta_{1}\right)-\sum_{k=\log _{q}(a)}^{\log _{q}(b)-1} q^{k}(q-1) \tilde{s}\left(q^{k}\right)\left[\left(\frac{\tilde{r}\left(q^{k}\right)}{\tilde{s}\left(q^{k}\right)}\right)^{2}-1\right]\right)
\end{aligned}
$$

Remark 4 In Example 9, we get some new inequalities involving the Csiszár divergence for quantum calculus.

Corollary 1 Under the conditions of Theorem 4 , if $m \geq 0$, then

$$
\begin{aligned}
0 & \leq \frac{1}{2} m\left[\left(\zeta_{2}-1\right)\left(1-\zeta_{1}\right)-D_{\chi^{2}}(\tilde{r}, \tilde{s})\right] \\
& \leq \frac{\left(\zeta_{2}-1\right)}{\zeta_{2}-\zeta_{1}} \Psi\left(\zeta_{1}\right)+\frac{\left(1-\zeta_{1}\right)}{\zeta_{2}-\zeta_{1}} \Psi\left(\zeta_{2}\right)-I_{\Psi}(\tilde{r}, \tilde{s}) .
\end{aligned}
$$

Proof We just need to show that

$$
D_{\chi^{2}}(\tilde{r}, \tilde{s}) \leq\left(\zeta_{2}-1\right)\left(1-\zeta_{1}\right)
$$

which follows from the proof of Theorem 3, since

$$
\begin{aligned}
0 & \leq \int_{a}^{b} \frac{\left(\zeta_{2} \tilde{s}(y)-\tilde{r}(y)\right)\left(\tilde{r}(y)-\zeta_{1} \tilde{s}(y)\right)}{\tilde{s}(y)} \Delta y \\
& =\left(\zeta_{2}-1\right)\left(1-\zeta_{1}\right)-D_{\chi^{2}}(\tilde{r}, \tilde{s}) .
\end{aligned}
$$

Example 10 Choosing $\mathbb{T}=\mathbb{R}$ in Corollary 1 , we get [7, Corollary 1 on p. 5].

\section{Bounds of some divergence measures}

First we recall some special means:

$$
\begin{aligned}
& A\left(\delta_{1}, \delta_{2}\right)=\frac{\delta_{1}+\delta_{2}}{2} \quad(\text { arithmetic mean }), \\
& G\left(\delta_{1}, \delta_{2}\right)= \pm \sqrt{\delta_{1} \delta_{2}} \quad \text { (geometric mean), } \\
& L\left(\delta_{1}, \delta_{2}\right)=\left\{\begin{array}{ll}
\delta_{2} & \text { if } \delta_{1}=\delta_{2}, \\
\frac{\delta_{2}-\delta_{1}}{\ln \delta_{2}-\ln \delta_{1}} & \text { if } \delta_{1} \neq \delta_{2}, \delta_{1}, \delta_{2}>0
\end{array} \quad\right. \text { (logarithmic mean) }
\end{aligned}
$$

and

$$
I\left(\delta_{1}, \delta_{2}\right)=\left\{\begin{array}{ll}
\delta_{2} & \text { if } \delta_{1}=\delta_{2} ; \\
\frac{1}{e}\left(\frac{\delta_{2}^{\delta_{2}}}{\delta_{1}^{\delta_{1}}}\right)^{\frac{1}{\delta_{2}-\delta_{1}}} & \text { if } \delta_{1} \neq \delta_{2}
\end{array} \quad\right. \text { (identric mean). }
$$




\subsection{Kullback-Leibler divergence on time scales}

Let $\psi:(0, \infty) \rightarrow \mathbb{R}$ be the convex mapping $\psi(t)=t \ln t$. Then

$$
I_{\psi}(\tilde{r}, \tilde{s})=\int_{a}^{b} \tilde{r}(y) \ln \left[\frac{\tilde{r}(y)}{\tilde{s}(y)}\right] \Delta y=D(\tilde{r}, \tilde{s}),
$$

where $D(\tilde{r}, \tilde{s})$ is the Kullback-Leibler distance.

\section{Proposition 1 If}

$$
\zeta_{1} \leq \frac{\tilde{r}(y)}{\tilde{s}(y)} \leq \zeta_{2} \quad \text { for all } y \in \mathbb{T},
$$

then

$$
D(\tilde{r}, \tilde{s})=\int_{a}^{b} \tilde{r}(y) \ln \left[\frac{\tilde{r}(y)}{\tilde{s}(y)}\right] \Delta y \leq \ln I\left(\zeta_{1}, \zeta_{2}\right)-\frac{G^{2}\left(\zeta_{1}, \zeta_{2}\right)}{L\left(\zeta_{1}, \zeta_{2}\right)}+1,
$$

where $G(\cdot, \cdot)$ is the usual geometric mean, $L(\cdot, \cdot)$ is the logarithmic mean, and $I(\cdot, \cdot)$ is the identric mean.

Proof Using Theorem 2 for $\psi(t)=t \ln t$, we obtain

$$
\begin{aligned}
D(\tilde{r}, \tilde{s}) & =\int_{a}^{b} \tilde{r}(y) \ln \left[\frac{\tilde{r}(y)}{\tilde{s}(y)}\right] \Delta y \leq \frac{\zeta_{2}-1}{\zeta_{2}-\zeta_{1}} \zeta_{1} \ln \zeta_{1}+\frac{1-\zeta_{1}}{\zeta_{2}-\zeta_{1}} \zeta_{2} \ln \zeta_{2} \\
& =\frac{\zeta_{1} \zeta_{2} \ln \zeta_{1}-\zeta_{1} \ln \zeta_{1}+\zeta_{2} \ln \zeta_{2}-\zeta_{1} \zeta_{2} \ln \zeta_{2}}{\zeta_{2}-\zeta_{1}} \\
& =\frac{\zeta_{2} \ln \zeta_{2}-\zeta_{1} \ln \zeta_{1}}{\zeta_{2}-\zeta_{1}}-\zeta_{1} \zeta_{2} \frac{\left[\ln \zeta_{2}-\ln \zeta_{1}\right]}{\zeta_{2}-\zeta_{1}} \\
& =\ln \left(\frac{\zeta_{2}^{\zeta_{2}}}{\zeta_{1}^{\zeta_{1}}}\right)^{\frac{1}{\zeta_{2}-\zeta_{1}}}-\ln e+\ln e-\left(\sqrt{\zeta_{1} \zeta_{2}}\right)^{2} \frac{\left[\ln \zeta_{2}-\ln \zeta_{1}\right]}{\zeta_{2}-\zeta_{1}} \\
& =\ln I\left(\zeta_{1}, \zeta_{2}\right)+1-\frac{G^{2}\left(\zeta_{1}, \zeta_{2}\right)}{L\left(\zeta_{1}, \zeta_{2}\right)}
\end{aligned}
$$

Example 11 Choosing $\mathbb{T}=\mathbb{R}$ in Proposition 1, we get [7, Proposition 1 on p. 6].

Example 12 Choosing $\mathbb{T}=h \mathbb{Z}, h>0$, in Proposition 1, we obtain

$$
\sum_{k=\frac{a}{h}}^{\frac{b}{h}-1} \tilde{r}(k h) h \ln \left[\frac{\tilde{r}(k h)}{\tilde{s}(k h)}\right] \leq \ln I\left(\zeta_{1}, \zeta_{2}\right)-\frac{G^{2}\left(\zeta_{1}, \zeta_{2}\right)}{L\left(\zeta_{1}, \zeta_{2}\right)}+1 .
$$

Remark 5 Equation (24) is an extension of the specific bound for the Kullback-Leibler divergence obtained by Lovričević et al. [15, Corollary 4.4].

Example 13 Choosing $\mathbb{T}=q^{\mathbb{N}_{0}}(q>1)$ in Proposition 1, we have

$$
\sum_{k=\log _{q}(a)}^{\log _{q}(b)-1} q^{k}(q-1) \tilde{r}\left(q^{k}\right) \ln \left[\frac{\tilde{r}\left(q^{k}\right)}{\tilde{s}\left(q^{k}\right)}\right] \leq \ln I\left(\zeta_{1}, \zeta_{2}\right)-\frac{G^{2}\left(\zeta_{1}, \zeta_{2}\right)}{L\left(\zeta_{1}, \zeta_{2}\right)}+1 .
$$


Remark 6 Equation (25) shows an upper bound for the Kullback-Leibler divergence, which is new in quantum calculus.

Proposition 2 Under the conditions of Proposition 1, we get

$$
\begin{aligned}
0 & \leq \ln I\left(\zeta_{1}, \zeta_{2}\right)-\frac{G^{2}\left(\zeta_{1}, \zeta_{2}\right)}{L\left(\zeta_{1}, \zeta_{2}\right)}+1-\int_{a}^{b} \tilde{r}(y) \ln \left[\frac{\tilde{r}(y)}{\tilde{s}(y)}\right] \Delta y \\
& \leq \frac{\left(\zeta_{2}-1\right)\left(1-\zeta_{1}\right)-D_{\chi^{2}}(\tilde{r}, \tilde{s})}{L\left(\zeta_{1}, \zeta_{2}\right)}
\end{aligned}
$$

where $D_{\chi^{2}}(\tilde{r}, \tilde{s})$ is defined in Theorem 3.

Proof Apply Theorem 3 for $\psi(t)=t \ln t$ :

$$
\frac{\psi^{\prime}\left(\zeta_{2}\right)-\psi^{\prime}\left(\zeta_{1}\right)}{\zeta_{2}-\zeta_{1}}=\frac{\ln \zeta_{2}-\ln \zeta_{1}}{\zeta_{2}-\zeta_{1}}=\frac{1}{L\left(\zeta_{1}, \zeta_{2}\right)}
$$

Example 14 Putting $\mathbb{T}=\mathbb{R}$ in Proposition 2, we get [7, Proposition 2 on p. 6].

Example 15 Choosing $\mathbb{T}=q^{\mathbb{N}_{0}}(q>1)$, in Proposition 2, we have

$$
\begin{aligned}
0 & \leq \ln I\left(\zeta_{1}, \zeta_{2}\right)-\frac{G^{2}\left(\zeta_{1}, \zeta_{2}\right)}{L\left(\zeta_{1}, \zeta_{2}\right)}+1-\sum_{k=\log _{q}(a)}^{\log _{q}(b)-1} q^{k}(q-1) \tilde{r}\left(q^{k}\right) \ln \left[\frac{\tilde{r}\left(q^{k}\right)}{\tilde{s}\left(q^{k}\right)}\right] \\
& \leq \frac{\left(\zeta_{2}-1\right)\left(1-\zeta_{1}\right)-\sum_{k=\log _{q}(a)}^{\log _{(}(b)-1} q^{k}(q-1) \tilde{r}\left(q^{k}\right)\left[\left(\frac{\tilde{s}\left(q^{k}\right)}{\tilde{r}\left(q^{k}\right)}\right)^{2}-1\right]}{L\left(\zeta_{1}, \zeta_{2}\right)} .
\end{aligned}
$$

By using Theorem 4 we can improve (26) as follows.

Proposition 3 Let $\tilde{r}, \tilde{s}$ satisfy (23). Then we have

$$
\begin{aligned}
& \frac{1}{2 \zeta_{2}}\left[\left(\zeta_{2}-1\right)\left(1-\zeta_{1}\right)-D_{\chi^{2}}(\tilde{r}, \tilde{s})\right] \\
& \quad \leq \ln I\left(\zeta_{1}, \zeta_{2}\right)-\frac{G^{2}\left(\zeta_{1}, \zeta_{2}\right)}{L\left(\zeta_{1}, \zeta_{2}\right)}+1-D(\tilde{r}, \tilde{s}) \\
& \quad \leq \frac{1}{2 \zeta_{1}}\left[\left(\zeta_{2}-1\right)\left(1-\zeta_{1}\right)-D_{\chi^{2}}(\tilde{r}, \tilde{s})\right] .
\end{aligned}
$$

Proof Consider $\psi(t)=t \ln t$ in Theorem 4. In this case, $\psi^{\prime \prime}(t)=\frac{1}{t}, t \in\left[\zeta_{1}, \zeta_{2}\right]$, and then

$$
\frac{1}{\zeta_{2}} \leq \psi^{\prime \prime}(t) \leq \frac{1}{\zeta_{1}}, \quad t \in\left[\zeta_{1}, \zeta_{2}\right]
$$

which gives the desired result.

Remark 7 For $\mathbb{T}=\mathbb{R}$ in Proposition 3, we get [7, Proposition 3 on p. 7]. 
Example 16 Choosing $\mathbb{T}=q^{\mathbb{N}_{0}}(q>1)$ in Proposition 3, we have

$$
\begin{aligned}
& \frac{1}{2 \zeta_{2}}\left(\left(\zeta_{2}-1\right)\left(1-\zeta_{1}\right)-\sum_{k=\log _{q}(a)}^{\log _{q}(b)-1} q^{k}(q-1) \tilde{r}\left(q^{k}\right)\left[\left(\frac{\tilde{s}\left(q^{k}\right)}{\tilde{r}\left(q^{k}\right)}\right)^{2}-1\right]\right) \\
& \quad \leq \ln I\left(\zeta_{1}, \zeta_{2}\right)-\frac{G^{2}\left(\zeta_{1}, \zeta_{2}\right)}{L\left(\zeta_{1}, \zeta_{2}\right)}+1-\sum_{k=\log _{q}(a)}^{\log _{q}(b)-1} q^{k}(q-1) \tilde{r}\left(q^{k}\right) \ln \left[\frac{\tilde{r}\left(q^{k}\right)}{\tilde{s}\left(q^{k}\right)}\right] \\
& \quad \leq \frac{1}{2 \zeta_{1}}\left(\left(\zeta_{2}-1\right)\left(1-\zeta_{1}\right)-\sum_{k=\log _{q}(a)}^{\log _{q}(b)-1} q^{k}(q-1) \tilde{r}\left(q^{k}\right)\left[\left(\frac{\tilde{s}\left(q^{k}\right)}{\tilde{r}\left(q^{k}\right)}\right)^{2}-1\right]\right) .
\end{aligned}
$$

Now consider the convex mapping $\psi(t)=-\ln t$. We get

$$
\begin{aligned}
I_{\psi}(\tilde{r}, \tilde{s}) & =-\int_{a}^{b} \tilde{s}(y) \ln \left[\frac{\tilde{r}(y)}{\tilde{s}(y)}\right] \Delta y \\
& =\int_{a}^{b} \tilde{s}(y) \ln \left[\frac{\tilde{s}(y)}{\tilde{r}(y)}\right] \Delta y=D(\tilde{s}, \tilde{r}) .
\end{aligned}
$$

By using Theorem 2 we obtain the following result.

Proposition 4 Let $\tilde{r}, \tilde{s}$ satisfy (23). Then

$$
D(\tilde{s}, \tilde{r})=\int_{a}^{b} \tilde{s}(y) \ln \left[\frac{\tilde{s}(y)}{\tilde{r}(y)}\right] \Delta y \leq \ln I\left(\frac{1}{\zeta_{1}}, \frac{1}{\zeta_{2}}\right)-\frac{1}{L\left(\zeta_{1}, \zeta_{2}\right)}+1 .
$$

Proof Using (1) for $\psi(t)=-\ln t$, we get

$$
\begin{aligned}
D(\tilde{s}, \tilde{r}) & =\int_{a}^{b} \tilde{s}(y) \ln \left[\frac{\tilde{s}(y)}{\tilde{r}(y)}\right] \Delta y \leq \frac{\left(\zeta_{2}-1\right)\left(-\ln \zeta_{1}\right)+\left(1-\zeta_{1}\right)\left(-\ln \zeta_{2}\right)}{\zeta_{2}-\zeta_{1}} \\
& =\frac{\left(\zeta_{1} \ln \zeta_{2}-\zeta_{2} \ln \zeta_{1}\right)}{\zeta_{2}-\zeta_{1}}-\frac{\left(\ln \zeta_{2}-\ln \zeta_{1}\right)}{\zeta_{2}-\zeta_{1}} \\
& =\frac{\zeta_{1} \zeta_{2}\left(\frac{1}{\zeta_{2}} \ln \zeta_{2}-\frac{1}{\zeta_{1}} \ln \zeta_{1}\right)}{\zeta_{2}-\zeta_{1}}-\frac{1}{L\left(\zeta_{1}, \zeta_{2}\right)} \\
& =\frac{\left(\frac{1}{\zeta_{1}} \ln \frac{1}{\zeta_{1}}-\frac{1}{\zeta_{2}} \ln \frac{1}{\zeta_{2}}\right)}{\frac{1}{\zeta_{1}}-\frac{1}{\zeta_{2}}}-\frac{1}{L\left(\zeta_{1}, \zeta_{2}\right)} \\
& =\ln I\left(\frac{1}{\zeta_{1}}, \frac{1}{\zeta_{2}}\right)-\frac{1}{L\left(\zeta_{1}, \zeta_{2}\right)}+1
\end{aligned}
$$

Remark 8 For $\mathbb{T}=\mathbb{R}$ in Proposition 4 , we get [7, Proposition 4 on p. 7].

Proposition 5 Let $\tilde{r}, \tilde{s}$ satisfy (23). Then we have

$$
\begin{aligned}
0 & \leq \ln I\left(\frac{1}{\zeta_{1}}, \frac{1}{\zeta_{2}}\right)-\frac{1}{L\left(\zeta_{1}, \zeta_{2}\right)}+1-\int_{a}^{b} \tilde{s}(y) \ln \left[\frac{\tilde{s}(y)}{\tilde{r}(y)}\right] \Delta y \\
& =\frac{1}{G^{2}\left(\zeta_{1}, \zeta_{2}\right)}\left[\left(\zeta_{2}-1\right)\left(1-\zeta_{1}\right)-D_{\chi^{2}}(\tilde{r}, \tilde{s})\right]
\end{aligned}
$$

where $D_{\chi^{2}}(\tilde{r}, \tilde{s})$ is defined in Theorem 3 . 
Proof Apply Theorem 3 for $\psi(t)=-\ln t$ :

$$
\frac{\psi^{\prime}\left(\zeta_{2}\right)-\psi^{\prime}\left(\zeta_{1}\right)}{\zeta_{2}-\zeta_{1}}=\frac{1}{\zeta_{1} \zeta_{2}}=\frac{1}{G^{2}\left(\zeta_{1}, \zeta_{2}\right)} .
$$

Remark 9 For $\mathbb{T}=\mathbb{R}$, Proposition 5 becomes [7, Proposition 5 on p. 7].

Further improvement of (27) is as follows.

Proposition 6 Under the assumptions of Theorem 4, we have

$$
\begin{aligned}
& \frac{1}{2 \zeta_{2}^{2}}\left[\left(\zeta_{2}-1\right)\left(1-\zeta_{1}\right)-D_{\chi^{2}}(\tilde{r}, \tilde{s})\right] \\
& \quad \leq \ln I\left(\frac{1}{\zeta_{1}}, \frac{1}{\zeta_{2}}\right)-\frac{1}{L\left(\zeta_{1}, \zeta_{2}\right)}+1-D(\tilde{s}, \tilde{r}) \\
& \quad \leq \frac{1}{2 \zeta_{1}^{2}}\left[\left(\zeta_{2}-1\right)\left(1-\zeta_{1}\right)-D_{\chi^{2}}(\tilde{r}, \tilde{s})\right] .
\end{aligned}
$$

Proof Apply Theorem 4, for which $\psi^{\prime \prime}(t)=\frac{1}{t^{2}}$ and

$$
\frac{1}{2 \zeta_{2}^{2}} \leq \psi^{\prime \prime}(t) \leq \frac{1}{2 \zeta_{1}^{2}}
$$

for all $t \in\left[\zeta_{1}, \zeta_{2}\right]$.

Remark 10 Choosing $\mathbb{T}=\mathbb{R}$ in Proposition 6, we get [7, Proposition 6 on p. 8].

\subsection{Triangular discrimination on time scales}

Let $\psi:[0, \infty) \rightarrow \mathbb{R}$ be the convex mapping $\psi(t)=\frac{(t-1)^{2}}{t+1}$. Then

$$
I_{\psi}(\tilde{r}, \tilde{s})=\int_{a}^{b} \frac{[\tilde{r}(y)-\tilde{s}(y)]^{2}}{\tilde{r}(y)+\tilde{s}(y)} \Delta y=D_{\Delta}(\tilde{r}, \tilde{s}),
$$

where $D_{\Delta}(\tilde{r}, \tilde{s})$ is the triangular discrimination.

Proposition 7 Under the assumptions of Theorem 2, we have

$$
D_{\Delta}(\tilde{r}, \tilde{s}) \leq \frac{4 A\left(\zeta_{1}, \zeta_{2}\right)-2 G^{2}\left(\zeta_{1}, \zeta_{2}\right)-2}{2 A\left(\zeta_{1}, \zeta_{2}\right)+G^{2}\left(\zeta_{1}, \zeta_{2}\right)+1}
$$

Proof Using Theorem 2 for $\psi(t)=\frac{(t-1)^{2}}{t+1}$, we obtain

$$
\begin{aligned}
& D_{\Delta}(\tilde{r}, \tilde{s}) \\
& \quad \leq \frac{\left(\zeta_{2}-1\right)\left(\zeta_{1}-1\right)^{2}\left(\zeta_{2}+1\right)+\left(1-\zeta_{1}\right)\left(\zeta_{2}-1\right)^{2}\left(\zeta_{1}+1\right)}{\left(\zeta_{2}-\zeta_{1}\right)\left(\zeta_{1}+1\right)\left(\zeta_{2}+1\right)} \\
& \quad=\frac{\zeta_{2}^{2} \zeta_{1}^{2}+\zeta_{2}^{2}-2 \zeta_{1} \zeta_{2}^{2}-\zeta_{1}^{2}-1+2 \zeta_{1}+\zeta_{2}^{2}+1-2 \zeta_{2}-\zeta_{2}^{2} \zeta_{1}^{2}-\zeta_{1}^{2}+2 \zeta_{2} \zeta_{1}^{2}}{\left(\zeta_{2}-\zeta_{1}\right)\left(\zeta_{1}+1\right)\left(\zeta_{2}+1\right)} \\
& \quad=\frac{\left(2 \zeta_{2}^{2}-2 \zeta_{1}^{2}\right)-\left(2 \zeta_{1} \zeta_{2}^{2}-2 \zeta_{2} \zeta_{1}^{2}\right)-\left(2 \zeta_{2}-2 \zeta_{1}\right)}{\left(\zeta_{2}-\zeta_{1}\right)\left(\zeta_{1}+1\right)\left(\zeta_{2}+1\right)}
\end{aligned}
$$




$$
\begin{aligned}
& =\frac{2\left(\zeta_{2}+\zeta_{1}\right)\left(\zeta_{2}-\zeta_{1}\right)-2 \zeta_{1} \zeta_{2}\left(\zeta_{2}-\zeta_{1}\right)-2\left(\zeta_{2}-\zeta_{1}\right)}{\left(\zeta_{2}-\zeta_{1}\right)\left(\zeta_{1}+1\right)\left(\zeta_{2}+1\right)} \\
& =\frac{2\left(\zeta_{2}+\zeta_{1}-\zeta_{1} \zeta_{2}-1\right)}{\left(\zeta_{1}+1\right)\left(\zeta_{2}+1\right)}=\frac{2\left(\zeta_{2}+\zeta_{1}-\zeta_{1} \zeta_{2}-1\right)}{\zeta_{1}+\zeta_{2}+\zeta_{1} \zeta_{2}+1} \\
& =\frac{4 A\left(\zeta_{1}, \zeta_{2}\right)-2 G^{2}\left(\zeta_{1}, \zeta_{2}\right)-2}{2 A\left(\zeta_{1}, \zeta_{2}\right)+G^{2}\left(\zeta_{1}, \zeta_{2}\right)+1} .
\end{aligned}
$$

The following example gives an upper bound for triangular discrimination, which is new in quantum calculus.

Example 17 Choosing $\mathbb{T}=q^{\mathbb{N}_{0}}(q>1)$ in Proposition 7, we have

$$
\sum_{k=\log _{q}(a)}^{\log _{q}(b)-1} q^{k}(q-1) \frac{\left[\tilde{r}\left(q^{k}\right)-\tilde{s}\left(q^{k}\right)\right]^{2}}{\tilde{r}\left(q^{k}\right)+\tilde{s}\left(q^{k}\right)} \leq \frac{4 A\left(\zeta_{1}, \zeta_{2}\right)-2 G^{2}\left(\zeta_{1}, \zeta_{2}\right)-2}{2 A\left(\zeta_{1}, \zeta_{2}\right)+G^{2}\left(\zeta_{1}, \zeta_{2}\right)+1}
$$

Proposition 8 Under the conditions of Theorem 3, we have

$$
\begin{aligned}
0 & \leq \frac{4 A\left(\zeta_{1}, \zeta_{2}\right)-2 G^{2}\left(\zeta_{1}, \zeta_{2}\right)-2}{2 A\left(\zeta_{1}, \zeta_{2}\right)+G^{2}\left(\zeta_{1}, \zeta_{2}\right)+1}-D_{\Delta}(\tilde{r}, \tilde{s}), \\
0 & \leq \frac{8 A\left(\zeta_{1}, \zeta_{2}\right)+8}{\left[G^{2}\left(\zeta_{1}, \zeta_{2}\right)+2 A\left(\zeta_{1}, \zeta_{2}\right)+1\right]^{2}}\left[\left(\zeta_{2}-1\right)\left(1-\zeta_{1}\right)-D_{\chi^{2}}(\tilde{r}, \tilde{s})\right] .
\end{aligned}
$$

Proof Apply Theorem 3 with $\psi(t)=\frac{(t-1)^{2}}{t+1}$, which gives $\psi^{\prime}(t)=1-\frac{4}{(1+t)^{2}}$ and

$$
\frac{\psi^{\prime}\left(\zeta_{2}\right)-\psi^{\prime}\left(\zeta_{1}\right)}{\zeta_{2}-\zeta_{1}}=\frac{4\left(\zeta_{2}+\zeta_{1}+2\right)}{\left[\left(\zeta_{2}+1\right)\left(\zeta_{1}+1\right)\right]^{2}}=\frac{8 A\left(\zeta_{1}, \zeta_{2}\right)+8}{\left[G^{2}\left(\zeta_{1}, \zeta_{2}\right)+2 A\left(\zeta_{1}, \zeta_{2}\right)+1\right]^{2}} .
$$

Proposition 9 Under the assumptions of Theorem 4, we have

$$
\begin{aligned}
0 & \leq \frac{8}{\left(1+\zeta_{2}\right)^{3}}\left[\left(\zeta_{2}-1\right)\left(1-\zeta_{1}\right)-D_{\chi^{2}}(\tilde{r}, \tilde{s})\right] \\
& \leq \frac{4 A\left(\zeta_{1}, \zeta_{2}\right)-2 G^{2}\left(\zeta_{1}, \zeta_{2}\right)-2}{2 A\left(\zeta_{1}, \zeta_{2}\right)+G^{2}\left(\zeta_{1}, \zeta_{2}\right)+1}-D_{\Delta}(\tilde{r}, \tilde{s}) \\
& \leq \frac{8}{\left(1+\zeta_{1}\right)^{3}}\left[\left(\zeta_{2}-1\right)\left(1-\zeta_{1}\right)-D_{\chi^{2}}(\tilde{r}, \tilde{s})\right] .
\end{aligned}
$$

Proof Use Theorem 4 for $\psi(t)=\frac{(t-1)^{2}}{t+1}$, which implies that $\psi^{\prime \prime}(t)=\frac{8}{(1+t)^{3}}$ and

$$
\frac{8}{\left(1+\zeta_{2}\right)^{3}} \leq \psi^{\prime \prime}(t) \leq \frac{8}{\left(1+\zeta_{1}\right)^{3}} \quad \text { for all } t \in\left[\zeta_{1}, \zeta_{2}\right] .
$$

\subsection{Hellinger discrimination on time scales}

Let $\psi:[0, \infty) \rightarrow \mathbb{R}$ be the convex mapping $\psi(t)=\frac{1}{2}(\sqrt{t}-1)^{2}$. Then

$$
\begin{aligned}
I_{\psi}(\tilde{r}, \tilde{s}) & =\frac{1}{2} \int_{a}^{b} \tilde{s}(y)\left(\sqrt{\frac{\tilde{r}(y)}{\tilde{s}(y)}}-1\right)^{2} \Delta y \\
& =\frac{1}{2} \int_{a}^{b}[\sqrt{\tilde{r}(y)}-\sqrt{\tilde{s}(y)}]^{2} \Delta y=h^{2}(\tilde{r}, \tilde{s}),
\end{aligned}
$$

where $h^{2}(\tilde{r}, \tilde{s})$ is the Hellinger discrimination. 
Proposition 10 Under the assumptions of Theorem 2, we get

$$
h^{2}(\tilde{r}, \tilde{s}) \leq \frac{2 A\left(\sqrt{\zeta_{1}}, \sqrt{\zeta_{2}}\right)-G\left(\zeta_{1}, \zeta_{2}\right)-1}{2 A\left(\sqrt{\zeta_{1}}, \sqrt{\zeta_{2}}\right)} .
$$

Proof Use Theorem 2 for $\psi(t)=\frac{1}{2}(\sqrt{t}-1)^{2}$ to obtain

$$
\begin{aligned}
& h^{2}(\tilde{r}, \tilde{s}) \\
& \quad \leq \frac{\left(\zeta_{2}-1\right) \frac{1}{2}\left(\sqrt{\zeta_{1}}-1\right)^{2}+\left(1-\zeta_{1}\right) \frac{1}{2}\left(\sqrt{\zeta_{2}}-1\right)^{2}}{\zeta_{2}-\zeta_{1}} \\
& \quad=\frac{\left(\frac{1}{2} \sqrt{\zeta_{2}}-1\right)\left(1-\sqrt{\zeta_{1}}\right)}{\zeta_{2}-\zeta_{1}}\left[\left(\sqrt{\zeta_{2}}+1\right)\left(1-\sqrt{\zeta_{1}}\right)+\left(1+\sqrt{\zeta_{1}}\right)\left(\sqrt{\zeta_{2}}-1\right)\right] \\
& \quad=\frac{\left(\sqrt{\zeta_{2}}-1\right)\left(1-\sqrt{\zeta_{1}}\right)\left(\sqrt{\zeta_{2}}-\sqrt{\zeta_{1}}\right)}{\zeta_{2}-\zeta_{1}}=\frac{\left(\sqrt{\zeta_{2}}-1\right)\left(1-\sqrt{\zeta_{1}}\right)}{\left(\sqrt{\zeta_{2}}+\sqrt{\zeta_{1}}\right)} \\
& =\frac{\sqrt{\zeta_{2}}+\sqrt{\zeta_{1}}-\sqrt{\zeta_{1} \zeta_{2}}-1}{\sqrt{\zeta_{1}}+\sqrt{\zeta_{2}}}=\frac{2 A\left(\sqrt{\zeta_{1}}, \sqrt{\zeta_{2}}\right)-G\left(\zeta_{1}, \zeta_{2}\right)-1}{2 A\left(\sqrt{\zeta_{1}}, \sqrt{\zeta_{2}}\right)} .
\end{aligned}
$$

Example 18 For $\mathbb{T}=\mathbb{R}$ in Proposition 10, we get [7, Proposition 7 on p. 8].

The following example gives an upper bound for the Hellinger discrimination, which is new in quantum calculus.

Example 19 Choosing $\mathbb{T}=q^{\mathbb{N}_{0}}(q>1)$ in Proposition 10, we have

$$
\sum_{k=\log _{q}(a)}^{\log _{q}(b)-1} q^{k}(q-1)\left[\sqrt{\tilde{r}\left(q^{k}\right)}-\sqrt{\tilde{s}\left(q^{k}\right)}\right]^{2} \leq \frac{2 A\left(\sqrt{\zeta_{1}}, \sqrt{\zeta_{2}}\right)-G\left(\zeta_{1}, \zeta_{2}\right)-1}{2 A\left(\sqrt{\zeta_{1}}, \sqrt{\zeta_{2}}\right)}
$$

Proposition 11 Under the assumptions of Theorem 3, we have

$$
\begin{aligned}
& 0 \leq \frac{\left(\sqrt{\zeta_{2}}-1\right)\left(1-\sqrt{\zeta_{1}}\right)}{\sqrt{\zeta_{2}}+\sqrt{\zeta_{1}}}-h^{2}(\tilde{r}, \tilde{s}), \\
& 0 \leq \frac{1}{4 \sqrt{\zeta_{1} \zeta_{2}} A\left(\sqrt{\zeta_{1}}, \sqrt{\zeta_{2}}\right)}\left[\left(\zeta_{2}-1\right)\left(1-\zeta_{1}\right)-D_{\chi^{2}}(\tilde{r}, \tilde{s})\right],
\end{aligned}
$$

where $A(\cdot, \cdot)$ is the arithmetic mean.

Proof Apply Theorem 3 with $\psi(t)=\frac{1}{2}(\sqrt{t}-1)^{2}$, which implies that $\psi^{\prime}(t)=\frac{1}{2}-\frac{1}{2 \sqrt{t}}$ and

$$
\frac{\psi^{\prime}\left(\zeta_{2}\right)-\psi^{\prime}\left(\zeta_{1}\right)}{\zeta_{2}-\zeta_{1}}=\frac{1}{2 \sqrt{\zeta_{1} \zeta_{2}}\left(\sqrt{\zeta_{2}}+\sqrt{\zeta_{1}}\right)}
$$

Remark 11 For $\mathbb{T}=\mathbb{R}$, Proposition 11 becomes [7, Proposition 8 on p. 9]. 
Proposition 12 Under the assumptions of Theorem 4, we have

$$
\begin{aligned}
0 & \leq \frac{1}{8 \sqrt{\zeta_{2}^{3}}}\left[\left(\zeta_{2}-1\right)\left(1-\zeta_{1}\right)-D_{\chi^{2}}(\tilde{r}, \tilde{s})\right] \\
& \leq \frac{\left(\sqrt{\zeta_{2}}-1\right)\left(1-\sqrt{\zeta_{1}}\right)}{\sqrt{\zeta_{2}}+\sqrt{\zeta_{1}}}-h^{2}(\tilde{r}, \tilde{s}) \\
& \leq \frac{1}{8 \sqrt{\zeta_{1}^{3}}}\left[\left(\zeta_{2}-1\right)\left(1-\zeta_{1}\right)-D_{\chi^{2}}(\tilde{r}, \tilde{s})\right] .
\end{aligned}
$$

Proof Use Theorem 4 for $\psi(t)=\frac{1}{2}(\sqrt{t}-1)^{2}$, which gives $\psi^{\prime \prime}(t)=\frac{1}{4 t^{\frac{3}{2}}}$ and, obviously,

$$
\frac{1}{4 \zeta_{2}^{\frac{3}{2}}} \leq \psi^{\prime \prime}(t) \leq \frac{1}{4 \zeta_{1}^{\frac{3}{2}}} \quad \text { for all } t \in\left[\zeta_{1}, \zeta_{2}\right]
$$

Remark 12 Choosing $\mathbb{T}=\mathbb{R}$ in Proposition 12, we get [7, Proposition 9 on p. 9].

\subsection{Jeffreys distance on time scales}

Let $\psi:(0, \infty) \rightarrow \mathbb{R}$ be the convex mapping $\psi(t)=(t-1) \ln (t)$. Then

$$
I_{\psi}(\tilde{r}, \tilde{s})=\int_{a}^{b}(\tilde{r}(y)-\tilde{s}(y)) \ln \left[\frac{\tilde{r}(y)}{\tilde{s}(y)}\right] \Delta y=D_{J}(\tilde{r}, \tilde{s}),
$$

where $D_{J}(\tilde{r}, \tilde{s})$ is the Jeffreys distance.

Proposition 13 Let $\tilde{r}, \tilde{s}$ satisfy (23). Then we get

$$
D_{J}(\tilde{r}, \tilde{s})=\int_{a}^{b}(\tilde{r}(y)-\tilde{s}(y)) \ln \left[\frac{\tilde{r}(y)}{\tilde{s}(y)}\right] \Delta y \leq \ln I\left(\zeta_{1}, \zeta_{2}\right)-\frac{G^{2}\left(\zeta_{1}, \zeta_{2}\right)+1}{L\left(\zeta_{1}, \zeta_{2}\right)}+\ln I\left(\frac{1}{\zeta_{1}}, \frac{1}{\zeta_{2}}\right)+2
$$

Proof Use Theorem 2 for $\psi(t)=(t-1) \ln t$ to get

$$
\begin{aligned}
D_{J}(\tilde{r}, \tilde{s}) & =\int_{a}^{b}(\tilde{r}(y)-\tilde{s}(y)) \ln \left[\frac{\tilde{r}(y)}{\tilde{s}(y)}\right] \Delta y \\
& \leq \frac{\zeta_{2}-1}{\zeta_{2}-\zeta_{1}}\left(\zeta_{1}-1\right) \ln \zeta_{1}+\frac{1-\zeta_{1}}{\zeta_{2}-\zeta_{1}}\left(\zeta_{2}-1\right) \ln \zeta_{2} \\
& =\frac{\left(\zeta_{2}-1\right)\left(\zeta_{1}-1\right) \ln \zeta_{1}-\left(1-\zeta_{1}\right)\left(\zeta_{2}-1\right) \ln \zeta_{2}}{\zeta_{2}-\zeta_{1}} \\
& =\frac{\zeta_{2} \ln \zeta_{2}-\zeta_{1} \ln \zeta_{1}}{\zeta_{2}-\zeta_{1}}-\zeta_{1} \zeta_{2} \frac{\left[\ln \zeta_{2}-\ln \zeta_{1}\right]}{\zeta_{2}-\zeta_{1}}+\frac{\zeta_{1} \ln \zeta_{2}-\zeta_{2} \ln \zeta_{1}}{\zeta_{2}-\zeta_{1}}-\frac{\left[\ln \zeta_{2}-\ln \zeta_{1}\right]}{\zeta_{2}-\zeta_{1}} \\
& =\ln \left(\frac{\zeta_{2}^{\zeta_{2}}}{\zeta_{1}^{\zeta_{1}}}\right)^{\frac{1}{\zeta_{2}-\zeta_{1}}}-\ln e+\ln e-\left(\zeta_{1} \zeta_{2}+1\right) \frac{\left[\ln \zeta_{2}-\ln \zeta_{1}\right]}{\zeta_{2}-\zeta_{1}}+\frac{\zeta_{1} \zeta_{2}\left(\frac{1}{\zeta_{2}} \ln \zeta_{2}-\frac{1}{\zeta_{1}} \ln \zeta_{1}\right)}{\zeta_{2}-\zeta_{1}} \\
& =\ln I\left(\zeta_{1}, \zeta_{2}\right)+1-\frac{\left[\left(\sqrt{\zeta_{1} \zeta_{2}}\right)^{2}+1\right]}{L\left(\zeta_{1}, \zeta_{2}\right)}+\frac{\left(\frac{1}{\zeta_{1}} \ln \frac{1}{\zeta_{1}}-\frac{1}{\zeta_{2}} \ln \frac{1}{\zeta_{2}}\right)}{\frac{1}{\zeta_{1}}-\frac{1}{\zeta_{2}}} \\
& =\ln I\left(\zeta_{1}, \zeta_{2}\right)-\frac{G^{2}\left(\zeta_{1}, \zeta_{2}\right)+1}{L\left(\zeta_{1}, \zeta_{2}\right)}+\ln I\left(\frac{1}{\zeta_{1}}, \frac{1}{\zeta_{2}}\right)+2 .
\end{aligned}
$$


The following example gives an upper bound for the Jeffreys distance, which is new in quantum calculus.

Example 20 Choosing $\mathbb{T}=q^{\mathbb{N}_{0}}(q>1)$ in Proposition 13, we have

$$
\begin{aligned}
& \sum_{k=\log _{q}(a)}^{\log _{q}(b)-1} q^{k}(q-1)\left(\tilde{r}\left(q^{k}\right)-\tilde{s}\left(q^{k}\right)\right) \ln \left[\frac{\tilde{r}\left(q^{k}\right)}{\tilde{s}\left(q^{k}\right)}\right] \\
& \quad \leq \ln I\left(\zeta_{1}, \zeta_{2}\right)-\frac{G^{2}\left(\zeta_{1}, \zeta_{2}\right)+1}{L\left(\zeta_{1}, \zeta_{2}\right)}+\ln I\left(\frac{1}{\zeta_{1}}, \frac{1}{\zeta_{2}}\right)+2 .
\end{aligned}
$$

Proposition 14 Under the assumptions of Theorem 3, we get

$$
\begin{aligned}
0 & \leq \ln I\left(\zeta_{1}, \zeta_{2}\right)-\frac{G^{2}\left(\zeta_{1}, \zeta_{2}\right)+1}{L\left(\zeta_{1}, \zeta_{2}\right)}+\ln I\left(\frac{1}{\zeta_{1}}, \frac{1}{\zeta_{2}}\right)+2-D_{J}(\tilde{r}, \tilde{s}) \\
& \leq\left[\frac{1}{G^{2}\left(\zeta_{1}, \zeta_{2}\right)}+\frac{1}{L\left(\zeta_{1}, \zeta_{2}\right)}\right]\left[\left(\zeta_{2}-1\right)\left(1-\zeta_{1}\right)-D_{\chi^{2}}(\tilde{r}, \tilde{s})\right] .
\end{aligned}
$$

Proof Apply Theorem 3 for $\psi(t)=(t-1) \ln t$, for which

$$
\frac{\psi^{\prime}\left(\zeta_{2}\right)-\psi^{\prime}\left(\zeta_{1}\right)}{\zeta_{2}-\zeta_{1}}=\frac{1}{\zeta_{1} \zeta_{2}}+\frac{\ln \zeta_{2}-\ln \zeta_{1}}{\zeta_{2}-\zeta_{1}}=\frac{1}{G^{2}\left(\zeta_{1}, \zeta_{2}\right)}+\frac{1}{L\left(\zeta_{1}, \zeta_{2}\right)} .
$$

Proposition 15 Under the assumptions of Theorem 4, we have

$$
\begin{aligned}
& \frac{\zeta_{2}+1}{\zeta_{2}^{2}}\left[\left(\zeta_{2}-1\right)\left(1-\zeta_{1}\right)-D_{\chi^{2}}(\tilde{r}, \tilde{s})\right] \\
& \quad \leq \ln I\left(\zeta_{1}, \zeta_{2}\right)-\frac{G^{2}\left(\zeta_{1}, \zeta_{2}\right)+1}{L\left(\zeta_{1}, \zeta_{2}\right)}+\ln I\left(\frac{1}{\zeta_{1}}, \frac{1}{\zeta_{2}}\right)+2-D_{J}(\tilde{r}, \tilde{s}) \\
& \quad \leq \frac{\zeta_{1}+1}{\zeta_{1}^{2}}\left[\left(\zeta_{2}-1\right)\left(1-\zeta_{1}\right)-D_{\chi^{2}}(\tilde{r}, \tilde{s})\right] .
\end{aligned}
$$

Proof Consider $\psi(t)=(t-1) \ln t$ in Theorem 4. In this case, $\psi^{\prime \prime}(t)=\frac{t+1}{t^{2}}, t \in\left[\zeta_{1}, \zeta_{2}\right]$, and then

$$
\frac{\zeta_{2}+1}{\zeta_{2}^{2}} \leq \psi^{\prime \prime}(t) \leq \frac{\zeta_{1}+1}{\zeta_{1}^{2}}, \quad t \in\left[\zeta_{1}, \zeta_{2}\right]
$$

which gives the desired result.

\subsection{Bhattacharyya distance on time scales}

Let $\psi:[0, \infty) \rightarrow \mathbb{R}$ be a convex mapping, $\psi(t)=-\sqrt{t}$. Then

$$
\begin{aligned}
I_{\psi}(\tilde{r}, \tilde{s}) & =-\int_{a}^{b} \tilde{s}(y) \sqrt{\frac{\tilde{r}(y)}{\tilde{s}(y)}} \Delta y \\
& =-\int_{a}^{b} \sqrt{\tilde{s}(y) \tilde{r}(y)} \Delta y=D_{B}(\tilde{r}, \tilde{s}),
\end{aligned}
$$

where $D_{B}(\tilde{r}, \tilde{s})$ is the Bhattacharyya distance. 
Proposition 16 Under the assumptions of Theorem 2, we obtain

$$
D_{B}(\tilde{r}, \tilde{s}) \leq \frac{-1-G\left(\zeta_{1}, \zeta_{2}\right)}{2 A\left(\sqrt{\zeta_{1}}, \sqrt{\zeta_{2}}\right)}
$$

Proof Use Theorem 2 for $\psi(t)=-\sqrt{t}$ to obtain

$$
\begin{aligned}
D_{B}(\tilde{r}, \tilde{s}) & \leq \frac{\left(\zeta_{2}-1\right)\left(-\sqrt{\zeta_{1}}\right)+\left(1-\zeta_{1}\right)\left(-\sqrt{\zeta_{2}}\right)}{\zeta_{2}-\zeta_{1}} \\
& =\frac{-\sqrt{\zeta_{2}}+\sqrt{\zeta_{1}}-\zeta_{2} \sqrt{\zeta_{1}}+\zeta_{1} \sqrt{\zeta_{2}}}{\zeta_{2}-\zeta_{1}} \\
& =\frac{-1\left(\sqrt{\zeta_{2}}-\sqrt{\zeta_{1}}\right)-\sqrt{\zeta_{1}} \sqrt{\zeta_{2}}\left(\sqrt{\zeta_{2}}-\sqrt{\zeta_{1}}\right)}{\left(\sqrt{\zeta_{2}}-\sqrt{\zeta_{1}}\right)\left(\sqrt{\zeta_{2}}+\sqrt{\zeta_{1}}\right)} \\
& =\frac{\left(-1-\sqrt{\zeta_{1}} \sqrt{\zeta_{2}}\right)}{\left(\sqrt{\zeta_{2}}+\sqrt{\zeta_{1}}\right)}=\frac{-1-G\left(\zeta_{1}, \zeta_{2}\right)}{2 A\left(\sqrt{\zeta_{1}}, \sqrt{\zeta_{2}}\right)} .
\end{aligned}
$$

Example 21 Choosing $\mathbb{T}=q^{\mathbb{N}_{0}}(q>1)$ in Proposition 16, we have

$$
\sum_{k=\log _{q}(a)}^{\log _{q}(b)-1}-\sqrt{q^{k}(q-1) \tilde{r}\left(q^{k}\right) \tilde{s}\left(q^{k}\right)} \leq \frac{-1-G\left(\zeta_{1}, \zeta_{2}\right)}{2 A\left(\sqrt{\zeta_{1}}, \sqrt{\zeta_{2}}\right)}
$$

Remark 13 In Example 21, we get an upper bound for the Bhattacharyya distance, which is new in quantum calculus.

Proposition 17 Under the assumptions of Theorem 3, we have

$$
\begin{aligned}
& 0 \leq \frac{-1-G\left(\zeta_{1}, \zeta_{2}\right)}{2 A\left(\sqrt{\zeta_{1}}, \sqrt{\zeta_{2}}\right)}-D_{B}(\tilde{r}, \tilde{s}), \\
& 0 \leq \frac{1}{4 G\left(\zeta_{1}, \zeta_{2}\right) A\left(\sqrt{\zeta_{1}}, \sqrt{\zeta_{2}}\right)}\left[\left(\zeta_{2}-1\right)\left(1-\zeta_{1}\right)-D_{\chi^{2}}(\tilde{r}, \tilde{s})\right],
\end{aligned}
$$

where $A(\cdot, \cdot)$ is the arithmetic mean.

Proof Apply Theorem 3 with $\psi(t)=-\sqrt{t}$, which implies that $\psi^{\prime}(t)=-\frac{1}{2 \sqrt{t}}$ and

$$
\frac{\psi^{\prime}\left(\zeta_{2}\right)-\psi^{\prime}\left(\zeta_{1}\right)}{\zeta_{2}-\zeta_{1}}=\frac{1}{2 \sqrt{\zeta_{1} \zeta_{2}}\left(\sqrt{\zeta_{2}}+\sqrt{\zeta_{1}}\right)}
$$

Proposition 18 Under the assumptions of Theorem 4, we have

$$
\begin{aligned}
0 & \leq \frac{1}{8 \sqrt{\zeta_{2}^{3}}}\left[\left(\zeta_{2}-1\right)\left(1-\zeta_{1}\right)-D_{\chi^{2}}(\tilde{r}, \tilde{s})\right] \\
& \leq \frac{-1-G\left(\zeta_{1}, \zeta_{2}\right)}{2 A\left(\sqrt{\zeta_{1}}, \sqrt{\zeta_{2}}\right)}-D_{B}(\tilde{r}, \tilde{s}) \\
& \leq \frac{1}{8 \sqrt{\zeta_{1}^{3}}}\left[\left(\zeta_{2}-1\right)\left(1-\zeta_{1}\right)-D_{\chi^{2}}(\tilde{r}, \tilde{s})\right] .
\end{aligned}
$$


Proof Use Theorem 4 for $\psi(t)=-\sqrt{t}$, which implies that $\psi^{\prime \prime}(t)=\frac{1}{4 t^{\frac{3}{2}}}$ and, obviously,

$$
\frac{1}{4 \zeta_{2}^{\frac{3}{2}}} \leq \psi^{\prime \prime}(t) \leq \frac{1}{4 \zeta_{1}^{\frac{3}{2}}} \quad \text { for all } t \in\left[\zeta_{1}, \zeta_{2}\right]
$$

\subsection{Harmonic distance on time scales}

Let $\psi:(0, \infty) \rightarrow \mathbb{R}$ be the convex mapping $\psi(t)=\frac{-2 t}{1+t}$. Then

$$
I_{\psi}(\tilde{r}, \tilde{s})=-\int_{a}^{b} \frac{2 \tilde{r}(y) \tilde{s}(y)}{\tilde{r}(y)+\tilde{s}(y)} \Delta y=D_{\mathrm{Ha}}(\tilde{r}, \tilde{s})
$$

where $D_{\mathrm{Ha}}(\tilde{r}, \tilde{s})$ is the harmonic distance.

Proposition 19 Under the assumptions of Theorem 2, we have

$$
D_{\mathrm{Ha}}(\tilde{r}, \tilde{s}) \leq \frac{-2}{1+2 A\left(\zeta_{1}, \zeta_{2}\right)+G^{2}\left(\zeta_{1}, \zeta_{2}\right)}
$$

Proof Use Theorem 2 for $\psi(t)=\frac{-2 t}{1+t}$ to get

$$
\begin{aligned}
D_{\mathrm{Ha}}(\tilde{r}, \tilde{s}) & \leq \frac{\zeta_{2}-1}{\zeta_{2}-\zeta_{1}} \frac{-2 \zeta_{1}}{1+\zeta_{1}}+\frac{1-\zeta_{1}}{\zeta_{2}-\zeta_{1}} \frac{-2 \zeta_{2}}{1+\zeta_{2}} \\
& =\frac{-2 \zeta_{1} \zeta_{2}+2 \zeta_{1}-2 \zeta_{2}+2 \zeta_{1} \zeta_{2}}{\left(\zeta_{2}-\zeta_{1}\right)\left(\zeta_{1}+1\right)\left(\zeta_{2}+1\right)} \\
& =\frac{-2\left(\zeta_{2}-\zeta_{1}\right)}{\left(\zeta_{2}-\zeta_{1}\right)\left(\zeta_{1}+1\right)\left(\zeta_{2}+1\right)} \\
& =\frac{-2}{\left(\zeta_{1}+1\right)\left(\zeta_{2}+1\right)}=\frac{-2}{1+2 A\left(\zeta_{1}, \zeta_{2}\right)+G^{2}\left(\zeta_{1}, \zeta_{2}\right)}
\end{aligned}
$$

The following example gives an upper bound for the harmonic distance, which is new in quantum calculus.

Example 22 Choosing $\mathbb{T}=q^{\mathbb{N}_{0}}(q>1)$ in Proposition 19, we obtain

$$
-\sum_{k=\log _{q}(a)}^{\log _{q}(b)-1} \frac{2 q^{k}(q-1) \tilde{r}\left(q^{k}\right) \tilde{s}\left(q^{k}\right)}{\tilde{r}\left(q^{k}\right)+\tilde{s}\left(q^{k}\right)} \leq \frac{-2}{1+2 A\left(\zeta_{1}, \zeta_{2}\right)+G^{2}\left(\zeta_{1}, \zeta_{2}\right)} .
$$

Proposition 20 Under the conditions of Theorem 3, we have

$$
\begin{aligned}
& 0 \leq \frac{-2}{1+2 A\left(\zeta_{1}, \zeta_{2}\right)+G^{2}\left(\zeta_{1}, \zeta_{2}\right)}-D_{\mathrm{Ha}}(\tilde{r}, \tilde{s}), \\
& 0 \leq \frac{8 A\left(\zeta_{1}, \zeta_{2}\right)+8}{\left[G^{2}\left(\zeta_{1}, \zeta_{2}\right)+2 A\left(\zeta_{1}, \zeta_{2}\right)+1\right]^{2}}\left[\left(\zeta_{2}-1\right)\left(1-\zeta_{1}\right)-D_{\chi^{2}}(\tilde{r}, \tilde{s})\right] .
\end{aligned}
$$

Proof Apply Theorem 3 with $\psi(t)=\frac{-2 t}{1+t}$, which implies that $\psi^{\prime}(t)=\frac{-1}{(1+t)^{2}}$ and

$$
\frac{\psi^{\prime}\left(\zeta_{2}\right)-\psi^{\prime}\left(\zeta_{1}\right)}{\zeta_{2}-\zeta_{1}}=\frac{\left(\zeta_{2}+\zeta_{1}+2\right)}{\left[\left(\zeta_{2}+1\right)\left(\zeta_{1}+1\right)\right]^{2}}=\frac{2 A\left(\zeta_{1}, \zeta_{2}\right)+2}{\left[G^{2}\left(\zeta_{1}, \zeta_{2}\right)+2 A\left(\zeta_{1}, \zeta_{2}\right)+1\right]^{2}} .
$$


Proposition 21 Under the assumptions of Theorem 4, we get

$$
\begin{aligned}
0 & \leq \frac{2}{\left(1+\zeta_{2}\right)^{3}}\left[\left(\zeta_{2}-1\right)\left(1-\zeta_{1}\right)-D_{\chi^{2}}(\tilde{r}, \tilde{s})\right] \\
& \leq \frac{-2}{1+2 A\left(\zeta_{1}, \zeta_{2}\right)+G^{2}\left(\zeta_{1}, \zeta_{2}\right)}-D_{\mathrm{Ha}}(\tilde{r}, \tilde{s}) \\
& \leq \frac{2}{\left(1+\zeta_{1}\right)^{3}}\left[\left(\zeta_{2}-1\right)\left(1-\zeta_{1}\right)-D_{\chi^{2}}(\tilde{r}, \tilde{s})\right] .
\end{aligned}
$$

Proof Use Theorem 4 for $\psi(t)=\frac{-2 t}{1+t}$, which implies that $\psi^{\prime \prime}(t)=\frac{2}{(1+t)^{3}}$ and

$$
\frac{2}{\left(1+\zeta_{2}\right)^{3}} \leq \psi^{\prime \prime}(t) \leq \frac{2}{\left(1+\zeta_{1}\right)^{3}} \quad \text { for all } t \in\left[\zeta_{1}, \zeta_{2}\right]
$$

\section{Conclusion}

In this paper, we introduced the Csiszár $f$-divergence on time scales and establish inequalities involving the Csiszár $f$-divergence on time scales. The obtained results are an extension of some known results in the literature and report new results in $h$-discrete calculus and quantum calculus.

\section{Acknowledgements}

The research of Josip Pečarić is supported by the Ministry of Education and Science of the Russian Federation (Agreement number 02.a03.21.0008).

\section{Funding}

There is no funding for this work.

\section{Availability of data and materials}

Data sharing is not applicable to this paper as no data sets were generated or analyzed during the current study.

\section{Competing interests}

The authors declare that they have no competing interests.

\section{Authors' contributions}

All authors jointly worked on the results and read and approved the final manuscript.

\section{Author details}

'Department of Mathematics, University of Sargodha, Sargodha, Pakistan. ${ }^{2}$ Department of Mathematics, University of Lahore (Sargodha Campus), Sargodha, Pakistan. ${ }^{3}$ Catholic University of Croatia, Zagreb, Croatia. ${ }^{4}$ RUDN University, Moscow, Russia.

\section{Publisher's Note}

Springer Nature remains neutral with regard to jurisdictional claims in published maps and institutional affiliations.

Received: 23 September 2020 Accepted: 3 December 2020 Published online: 10 December 2020

\section{References}

1. Agarwal, R., Bohner, M., Peterson, A.: Inequalities on time scales: a survey. Math. Inequal. Appl. 4, 535-557 (2001)

2. Ansari, I., Khan, K.A., Noesheen, A., Pečarić, Đ., Pečarić, J.: Shannon type inequalities via time scales theory. Adv. Differ. Equ. 2020, 135 (2020)

3. Anwar, M., Bibi, R., Bohner, M., Pečarić, J.: Integral inequalities on time scales via the theory of isotonic linear functionals. Abstr. Appl. Anal. 2011, Article ID 483595 (2011)

4. Bohner, M., Peterson, A.: Dynamic Equations on Time Scales. Birkhäuser, Boston (2001)

5. Bohner, M., Peterson, A.: Advances in Dynamic Equations on Time Scales. Birkhäuser, Boston (2003)

6. Csiszár, I.: Information-type measures of difference of probability distributions and indirect observations. Studia Sci. Math. Hung. 2, 299-318 (1967)

7. Dragomir, S.S.: Other inequalities for Csiszár divergence and applications. Preprint, RGMIA Res. Rep. Coll. (2000)

8. Dragomir, S.S.: An upper bound for the Csiszár $f$-divergence in terms of the variational distance and applications. Panam. Math. J. 12(4), 43-54 (2002)

9. Gibbs, A.L.: On choosing and bounding probability metrics. Int. Stat. Rev. 70(3), 419-435 (2002) 
10. Guseinov, S.G.: Integration on time scales. J. Math. Anal. Appl. 285(1), 107-127 (2003)

11. Horváth, L., Pečarić, Đ., Pečarić, J.: Estimations of $f$ - and Rényi divergences by using a cyclic refinement of the Jensen's inequality. Bull. Malays. Math. Sci. Soc. 42(3), 933-946 (2019)

12. Khan, M.A., Pečarić, Đ., Pečarić, J.: On Zipf-Mandelbrot entropy. J. Comput. Appl. Math. 346 192-204 (2019)

13. Liese, F., Vajda, I.: Convex Statistical Distances. Teubner, Leipzig (1987)

14. Liu, H., Meng, F.: Nonlinear retarded integral inequalities on time scales and their applications. J. Math. Inequal. 12, 219-234 (2018)

15. Lovricevic, N., Pečarić, Đ., Pečarić, J.: Zipf-Mandelbrot law and superadditivity of the Jensen functional. Inequalities and Zipf-Mandelbrot Law, 61

16. Ma, Q.H., Wang, J.W., Ke, X.H., Pečarić, J.: On the boundedness of a class of nonlinear dynamic equations of second order. Appl. Math. Lett. 26(11), 1099-1105 (2013)

17. Saker, S.H.: Some nonlinear dynamic inequalities on time scales. Math. Inequal. Appl. 14(3), 633-645 (2011)

18. Sason, I., Verdú, S.: $f$-Divergence inequalities. IEEE Trans. Inf. Theory 62(11), 5973-6006 (2016)

19. Sun, Y.G., Hassan, T.: Some nonlinear dynamic integral inequalities on time scales. Appl. Math. Comput. 220(4), 221-225 (2013)

20. Vajda, I.: Theory of Statistical Inference and Information. Kluwer Academic, Boston (1989)

\section{Submit your manuscript to a SpringerOpen ${ }^{\circ}$} journal and benefit from:

- Convenient online submission

- Rigorous peer review

- Open access: articles freely available online

- High visibility within the field

- Retaining the copyright to your article

Submit your next manuscript at $\gg$ springeropen.com 